\title{
含全氟环丁基芳基醚结构的疏水性聚碳酸酯的合成及性能研究
}

\author{
胡丹 $a$ 赵昆峰 $a, b, c$ 童 琴*,a,b 何丹农*,a,b,c \\ ( ${ }^{a}$ 纳米技术及应用国家工程研究中心 上海 200241) \\ ( ${ }^{b}$ 上海交通大学材料科学与工程学院 上海 200240) \\ ( ${ }^{c}$ 上海健康医学院 上海 201318)
}

\begin{abstract}
摘要 以商业化的对羟基苯甲醚为原料，合成了一种新型的含有全氟环丁基芳基醚结构的疏水性聚碳酸酯. 该聚合物 在不降低材料透明性的前提下, 表现出了优异的热稳定性和疏水性, 其热分解温度为 $351{ }^{\circ} \mathrm{C}$, 涂膜与水的接触角为 $110^{\circ}$. 为了进一步探究全氟环丁基芳基醚结构对聚碳酸酯的性能影响，以含有全氟环丁基芳基醚结构的单体和双酚 $\mathrm{A}$ 单体为原料, 合成了一系列具有不同全氟环丁基芳基醚基团含量的聚碳酸酯. 通过性能比较可知, 将全氟环丁基芳基 醚结构引入聚碳酸酯, 能够降低聚碳酸酯的分散度, 提高其热稳定性和疏水性.
\end{abstract}

关键词 聚碳酸酯; 疏水性; 全氟环丁基芳基醚

\section{Synthesis and Properties of Perfluorocyclobutyl Aryl Ether-Based Hydrophobic Polycarbonate}

\author{
Hu, Dan ${ }^{a}$ Zhao, Kunfeng ${ }^{a, b, c}$ Tong, Qin*,a,b He, Dannong*,a,b,c \\ ( ${ }^{a}$ National Engineering Research Center for Nanotechnology, Shanghai 200241) \\ $\left({ }^{b}\right.$ School of Material Science and Engineering, Shanghai Jiao Tong University, Shanghai 200240) \\ ( Shanghai University of Medicine \& Health Sciences, Shanghai 201318)
}

\begin{abstract}
A novel hydrophobic polycarbonate containing perfluorocyclobutyl (PFCB) aryl ether was synthesized from commercially available $p$-methoxyphenol. The polymer exhibited excellent thermal stability and hydrophobicity without reducing the transparency of the material. The thermal decomposition temperature was $351{ }^{\circ} \mathrm{C}$ and the contact angle between the film and water was $110^{\circ}$. In order to further explore the effect of PFCB aryl ether structure on the properties of polycarbonate, a series of polycarbonates with different PFCB aryl ether group contents were synthesized from PFCB aryl ether monomer and bisphenol A monomer. By comparing their properties, the introduction of PFCB aryl ether structure into polycarbonate can reduce its dispersion and improve its thermal stability and hydrophobicity.

Keywords polycarbonate; hydrophobicity; perfluorocyclobutyl aryl ether
\end{abstract}

聚碳酸酯(PC) 是分子链中含有碳酸酯基的高分子 聚合物, 一种性能优良的工程塑料, 具有突出的冲击韧 性、透明性和尺寸稳定性, 优良的力学强度和电绝缘性, 使用温度范围宽, 在电子电器、汽车工业、航空航天等 领域得到广泛的应用 ${ }^{[1 \sim 3]}$. 但聚碳酸酯材料还存在着一 些不足之处, 主要缺点是材料的耐水解性能差, 分子链 中存在对水和热都比较敏感的碳酸酯键, 在高温有氧及 潮湿的环境下聚碳酸酯的分子链会发生不同程度的降 解, 导致材料被破坏, 严重制约了其广泛应用. 目前国
内外主要通过在聚碳酸酯表面涂覆耐水解及耐候性功 能涂层的手段来改善其性能 ${ }^{[4,5]}$. 例如将改性二氧化硅 溶胶及锆溶胶等涂覆于经过浓酸处理过的 PC 薄膜表面, 制备得到硬度和透明度高、疏水性强和耐候性高的涂层 薄膜 ${ }^{[6]}$, 从而具有很好的环境适应性, 但该方法工艺流 程复杂, 对设备的要求较高, 不适用于大规模的工业化 生产. 聚合物改性的另一重要方向是材料表面的超疏水 改性 ${ }^{[710]}$, 如采用氧气等离子体刻蚀和表面氟化处理的 方法来制备 PC 的超疏水表面，但经过磨损测试后，PC

\footnotetext{
* Corresponding authors.hdn_nercn@163.com; tongqin_7557@163.com

Received December 28, 2018; revised March 26, 2019; published online April 11, 2019.

Project supported by the Major National Scientific Research Projects (No. 2015CB931902) and the Shanghai Science and Technology Development Funds (No. 16QB1402200).

国家重大科学研究计划(No. 2015CB931902)和上海市青年科技启明星计划(B 类)(No. 16QB1402200)资助项目.
} 
表面的含 $\mathrm{F}$ 量下降, 疏水性纳米层薄膜受到破坏, 材料 的超疏水特性丧失 ${ }^{[11]}$. 因此，制备具有一定稳定性的疏 水性聚碳酸酯材料, 成为研究的重要方向. 将氟原子引 入到聚合物中, 可以使材料的表面能降低, 疏水性增强, 耐热性提高 ${ }^{[12]}$, 但含氟聚碳酸酯的疏水性仍有待提高.

全氟环丁基(perfluorocyclobutyl, PFCB)芳基醚是由 三氟乙烯基芳基醚在加热的条件下发生 $[2 \pi+2 \pi]$ 成环反 应所形成 ${ }^{[13]}$, 具有一种特殊的氟原子组成形式. 全氟环 丁基可看作是被 “弯折” 的全氟烷基链, 因此具有类似 于全氟烷基链的疏水性, 芳基可以提供较高的热分解温 度, 提高热稳定性, 同时全氟环丁基与芳氧基之间存在 无规则顺反构型, 可阻碍高分子链段的有序排列, 赋予 材料高透光性和低介电常数等性质 ${ }^{[14 \sim 18]}$. 鉴于全氟环 丁基芳基醚结构的优异性能，我们设计合成了含有全氟 环丁基芳基醚结构的双酚单体 PFCB2OH, 并将其与三 光气反应合成聚碳酸酯(PFCBPC)(图 1). 为了进一步研 究全氟环丁基芳基醚基团的引入对聚碳酸酯性能的影 响, 我们以不同比例的 $\mathrm{PFCB} 2 \mathrm{OH}$ 和双酚 $\mathrm{A}$ 单体为原料, 与三光气反应制备一系列聚碳酸酯材料, 并进行性能比 较. 研究结果显示, 全氟环丁基芳基醚结构的引入可以 提高聚碳酸酯材料的热稳定性和疏水性.

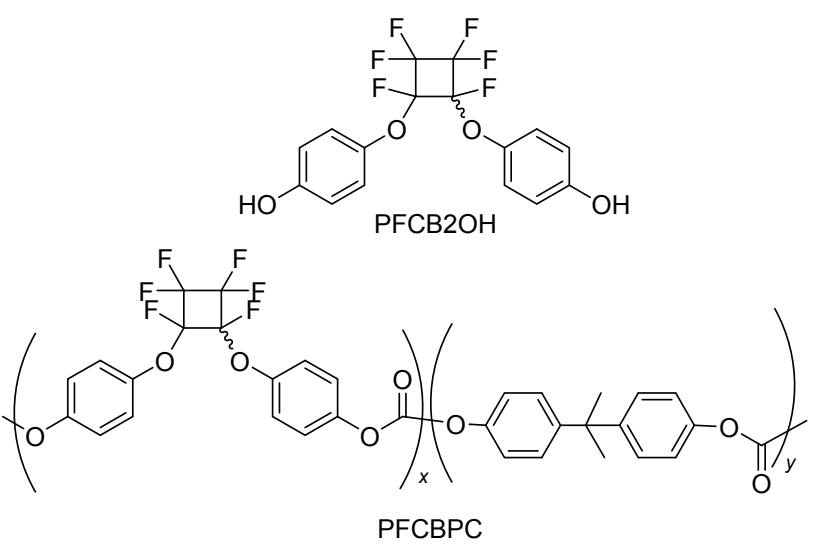

图 1 单体 $\mathrm{PFCB} 2 \mathrm{OH}$ 的化学结构

Figure 1 Chemical structure of monomer PFCB2OH

\section{1 结果与讨论}

\section{1 聚碳酸酯单体的合成与表征}

单体 PFCB2OH 的合成路线如 Scheme 1 所示, 以商 业化的对羟基苯甲醚为前体, 首先与 1,2-二溴四氟乙烷 在无水的条件下进行亲核取代反应生成 2-溴四氟乙烯 氧基苯甲醚类中间体. 其中 $\mathrm{CsCO}_{3}$ 既充当了干燥剂的作 用, 又充当了碱的作用, 之后在锌粉作用下经过脱卤化 反应得到 4-(三氟乙烯氧基)苯甲醚, 在加热条件下进行 $[2 \pi+2 \pi]$ 成环反应得到二聚中间体(PFCB2OMe $)^{[13]}$, 最 后经 $\mathrm{BBr}_{3}$ 脱除甲氧基得到含全氟环丁基芳基醚结构的
单体(PFCB2OH).
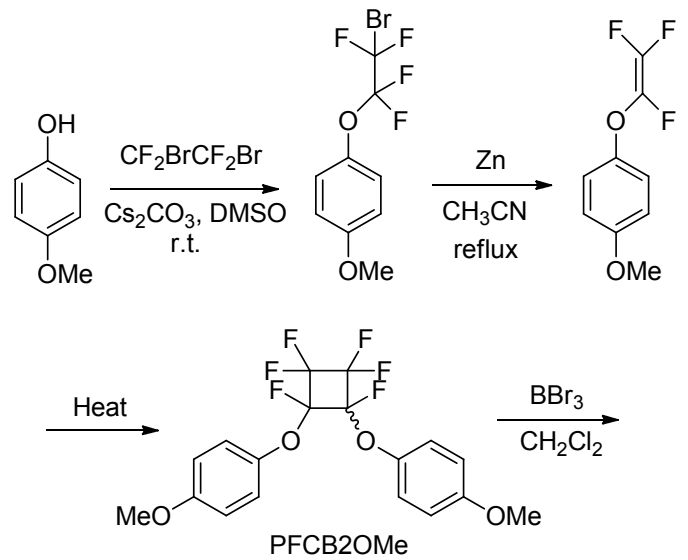<smiles>Oc1ccc(OC2(F)C(F)(F)C(F)(F)C2(F)Oc2ccc(O)cc2)cc1</smiles>

图式 1 聚碳酸酯单体 PFCB2OH 的合成路径

Scheme 1 Synthesis of the polycarbonate monomer PFCB2OH

通过 ${ }^{1} \mathrm{H}$ NMR, ${ }^{19} \mathrm{~F}$ NMR, FT-IR 等谱图对单体 $\mathrm{PFCB} 2 \mathrm{OH}$ 的化学结构进行了表征. 图 2 为单体 $\mathrm{PFCB} 2 \mathrm{OH}$ 的核磁氢谱, $\delta 6.79$ 处的 $\mathrm{c}$ 峰归属于苯环上羟 基间位质子峰, $\delta 7.03$ 处的 $\mathrm{b}$ 峰归属于苯环上羟基邻位 质子峰 ${ }^{[19]}, \delta 9.64$ 处的 $\mathrm{a}$ 峰归属于羟基的质子峰. 从 $\mathrm{PFCB} 2 \mathrm{OH}$ 的 ${ }^{19} \mathrm{~F} \mathrm{NMR}$ 谱图(图 3)中可以看到, PFCB 的 特征峰出现在 $\delta-128.08 \sim-132.51$ 之间 ${ }^{[20,21]}$. 图 4 为 $\mathrm{PFCB} 2 \mathrm{OH}$ 单体的红外谱图, 其中 $961 \mathrm{~cm}^{-1}$ 为 $\mathrm{PFCB}$ 的 特征吸收峰 ${ }^{[22]}$. 以上谱图结果证明了制备所得产物的 化学结构与目标产物相符.

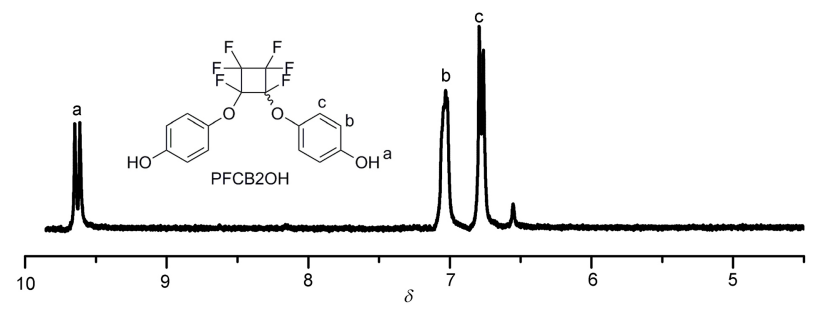

图 2 单体 $\mathrm{PFCB} 2 \mathrm{OH}$ 的核磁共振氢谱谱图

Figure $2{ }^{1} \mathrm{H}$ NMR spectrum of PFCB2OH (DMSO- $d_{6} 400$ $\mathrm{MHz})$

\section{2 聚碳酸酯的合成与表征}

在成功制备得到 $\mathrm{PFCB} 2 \mathrm{OH}$ 单体后, 我们以三光气 作为偶联剂, 以单体 $\mathrm{PFCB} 2 \mathrm{OH}$ 为原料, 三乙胺为缚酸 剂, 制备得到白色固体, 即主链含有全氟环丁基芳基醚 结构的聚碳酸酯 PFCBPC, 具体合成过程如 Eq. 1 所示. 


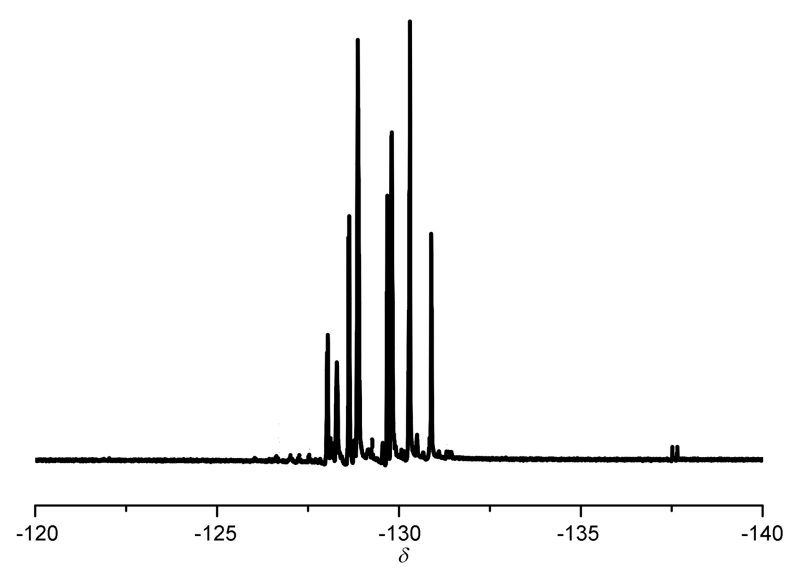

图 3 单体 $\mathrm{PFCB} 2 \mathrm{OH}$ 的核磁氟谱谱图

Figure $3{ }^{19} \mathrm{~F}$ NMR spectrum of PFCB2OH (DMSO- $d_{6} 400$ $\mathrm{MHz})$

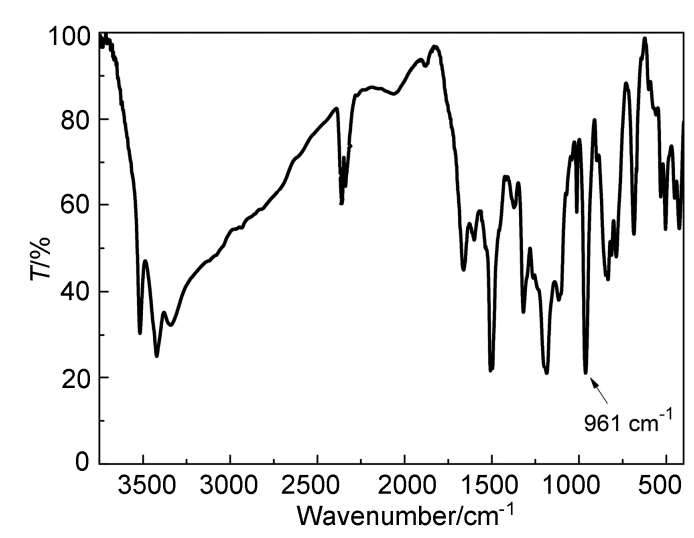

图 4 单体 $\mathrm{PFCB} 2 \mathrm{OH}$ 的红外谱图

Figure 4 FT-IR spectrum of PFCB2OH

通过 ${ }^{1} \mathrm{H}$ NMR, ${ }^{19} \mathrm{~F}$ NMR, FT-IR 和 GPC 等手段对合成的 聚合物进行表征. 图 5 为聚合物 PFCBPC 的核磁氢谱, $\delta 7.11 \sim 7.25$ 处的特征峰归属于苯环上羟基邻间位质子 峰. 从 PFCBPC 的 ${ }^{19} \mathrm{~F}$ NMR 谱图(图 6)中, 可以看到在 $-126.39 \sim-132.20$ 之间出现明显的特征峰, 证明了全 氟环丁基芳基醚结构的存在. 图 7 为 PFCBPC 的红外谱 图, $3060 \mathrm{~cm}^{-1}$ 处的吸收峰对应于苯环碳氢伸缩振动, $1780 \mathrm{~cm}^{-1}$ 处的吸收峰对应于苯环羰基的伸缩振动, $1600,1500,833 \mathrm{~cm}^{-1}$ 处的吸收峰对应于 1,4-二取代苯环 的骨架振动, $1166 \mathrm{~cm}^{-1}$ 处的吸收峰对应于酯键的碳氧 伸缩振动, $961 \mathrm{~cm}^{-1}$ 处的吸收峰对应于 PFCB 环的特征 吸收峰, 其中 $2359 \mathrm{~cm}^{-1}$ 对应于空气中 $\mathrm{CO}_{2}$ 的吸收峰. 以上表征结果确定了聚合物 PFCBPC 的化学结构. 通过 GPC 测得合成聚合物的数均分子量为 12500 , 分散度为 1.33. 全氟环丁基芳基醚结构中无规分布的顺式和反式 构型对聚合物 PFCBPC 的溶解性具有很大贡献, 其良好 的溶解性使得 PFCBPC 能够进行溶液加工, 同时由于全 氟环丁基芳基醚结构的引入, 赋予聚碳酸酯良好的柔
性，减小了聚合物分子的应力缺陷，提高了其加工性能.<smiles>[Y]c1ccc(OC2(OC3=CC=C(O)[C+]=C3O)C(F)(F)C(F)(F)C2(F)F)cc1</smiles><smiles>CCN(CC)C(=O)OC(Cl)(Cl)Cl</smiles><smiles>CC(C)Oc1ccc(OC(=O)C(C)(C)C)cc1</smiles>

PFCBPC

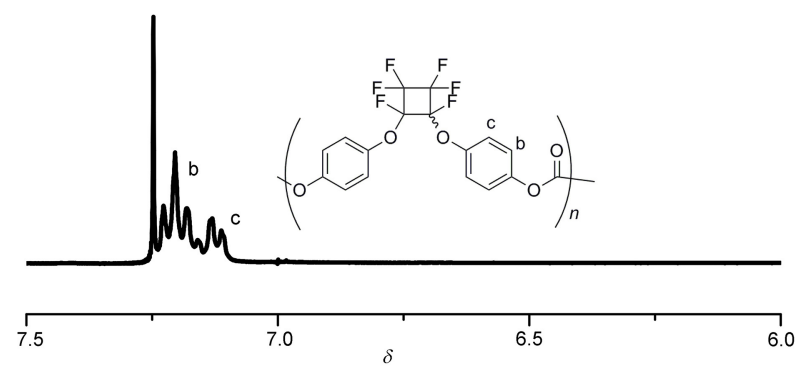

图 5 聚合物 PFCBPC 的核磁共振氢谱谱图 Figure $5{ }^{1} \mathrm{H}$ NMR spectrum of PFCBPC $\left(\mathrm{CDCl}_{3} 400 \mathrm{MHz}\right)$

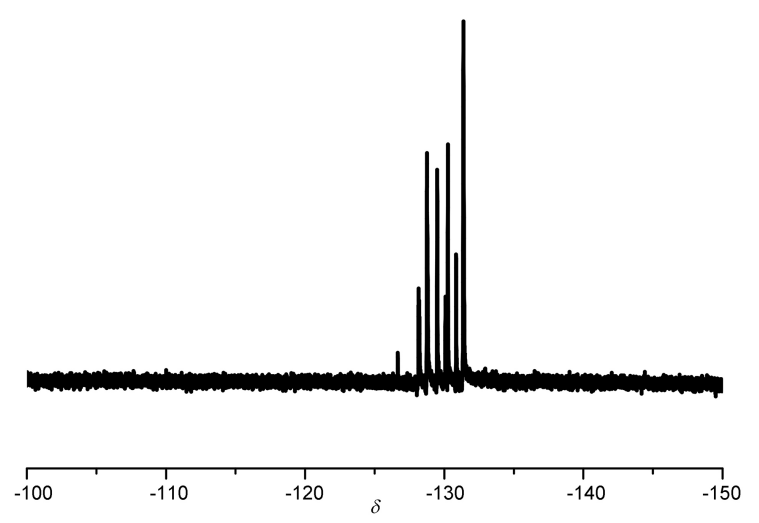

图 6 聚合物 PFCBPC 的核磁共振氟谱谱图

Figure $6{ }^{19} \mathrm{~F}$ NMR spectrum of PFCBPC $\left(\mathrm{CDCl}_{3} 400 \mathrm{MHz}\right)$

为了进一步探究全氟环丁基芳基醚结构对聚合物 材料的性能影响, 以不同比例的 $\mathrm{PFCB} 2 \mathrm{OH}$ 单体和双酚 $\mathrm{A}$ 单体为原料, 合成了一系列具有不同全氟环丁基芳基 醚基团含量的聚碳酸酯材料，合成方法与 PFCBPC 类似 (Eq. 1), 并对合成的聚合物进行了表征. 由 GPC 测得合 成聚合物材料的数均分子量和分散度，具体结果见于表 1. 从表 1 结果可以看出, 不同单体比例合成的聚碳酸酯 的数均分子量在同一级别, 且随着单体 $\mathrm{PFCB} 2 \mathrm{OH}$ 投料 


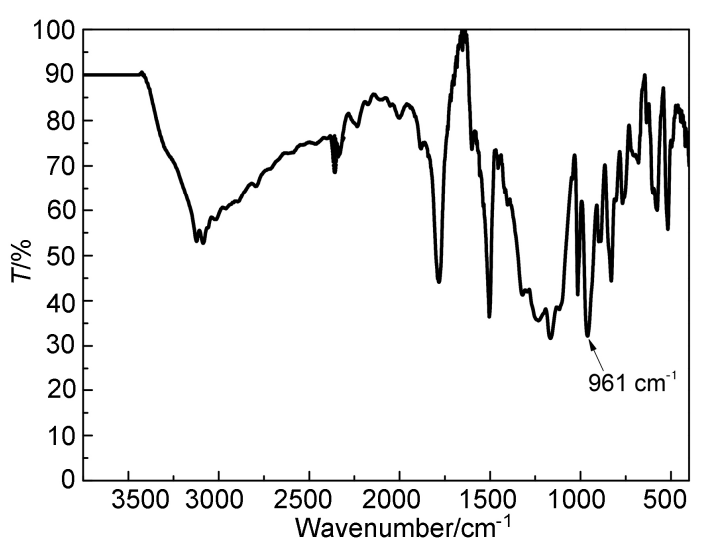

图 7 聚合物 PFCBPC 的红外谱图

Figure 7 FT-IR spectrum of PFCBPC

比例的增大, 聚碳酸酯的分散度逐渐降低, 分子量的分 布越来越均匀.

表 1 聚碳酸酯的数均分子量

Table 1 The average molecular weight of polycarbonates

\begin{tabular}{cccc}
\hline Sample & {$[\mathrm{PFCB} 2 \mathrm{OH}]:[\mathrm{BPA}]$} & $M_{n}$ & $M_{w} / M_{n}$ \\
\hline PFCBPC & $1: 0$ & 12500 & 1.33 \\
PFCBPC1 & $1: 1$ & 11600 & 1.37 \\
PFCBPC2 & $1: 2$ & 16200 & 1.41 \\
PFCBPC3 & $1: 3$ & 15400 & 1.43 \\
PC & $0: 1$ & 12500 & 1.46 \\
\hline
\end{tabular}

\section{3 聚碳酸酯的热稳定性和疏水性}

为了研究全氟环丁基芳基醚结构对聚碳酸酯性能 的影响, 对不同单体比例合成的聚碳酸酯的热稳定性及 疏水性进行了探究. 首先采用热重分析(TGA)对聚碳酸 酯的热稳定性进行了考察, 具体结果如表 2 所示. 从表 2 的 TGA 数据可以看到, 只以 $\mathrm{PFCB} 2 \mathrm{OH}$ 单体为原料合 成的聚碳酸酯 (PFCBPC) 的分解温度最高, 达到了 $351{ }^{\circ} \mathrm{C}$, 且 $5 \%$ 质量热重损失温度为 $392{ }^{\circ} \mathrm{C}, 10 \%$ 质量热 重损失温度为 $412{ }^{\circ} \mathrm{C}$, 展示了优异的热稳定性. 此外可 以看出, 随着聚合物单体中 $\mathrm{PFCB} 2 \mathrm{OH}$ 单体含量的增加, 聚碳酸酯的热分解温度基本呈逐渐增高的趋势, 热稳定 性也逐渐提高，说明全氟环丁基芳基醚结构的引入会提 高聚碳酸酯的热稳定性. 此外, 我们对合成聚碳酸酯材 料进行了透光率测试, 由紫外可见光谱的透过率数据
(表 2)可以看出，聚碳酸酯均具有很高的可见光透过率， 全氟环丁基芳基醚结构的引入对聚合物的透明性没有 影响.

通过测试材料与去离子水的接触角对合成聚碳酸 酯的疏水性进行了探究，实验结果见表 2. 从表 2 可以 看出, 水滴在 PFCBPC 薄膜上的接触角为 $110^{\circ}$, 传统的 以双酚 $\mathrm{A}(\mathrm{BPA})$ 为单体合成的聚碳酸酯 $(\mathrm{PC})$ 薄膜与水的 接触角为 $50^{\circ}$ (如图 8 所示), 说明全氟环丁基芳基醚结构 的引入会显著增大聚碳酸酯表面的疏水性能. 此外, 随 着聚合物单体中 $\mathrm{PFCB} 2 \mathrm{OH}$ 单体含量的增加, 聚碳酸酯 薄膜与水的接触角逐渐增大, 即聚碳酸酯的疏水性逐渐 提高，证明了全氟环丁基芳基醚结构对聚碳酸酯材料表 面性质的影响. 全氟基团具有较低的表面能，易于形成 氟碳相，具有向材料表面迁移的趋势，从而能够降低材

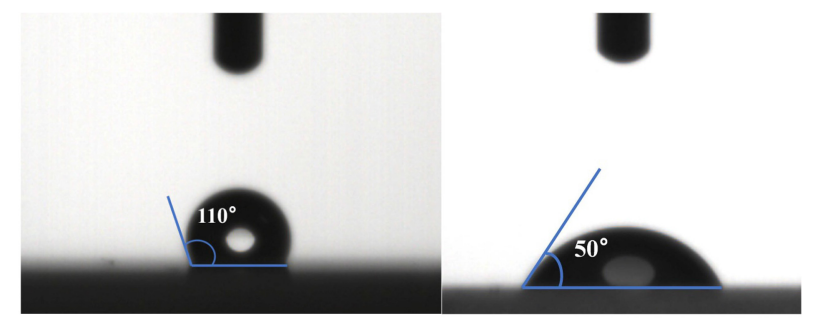

图 $8 \mathrm{PFCBPC}$ 和 $\mathrm{PC}$ 薄膜表面与水的接触角

Figure 8 Contact angle of water on PFCBPC and PC films

料表面处薄膜与空气的界面能来提高材料的疏水性能

\section{2 结论}

合成了具有全氟环丁基芳基醚结构的单体(PFCB$2 \mathrm{OH})$, 并将其与三光气反应合成聚碳酸酯 PFCBPC，成 功将全氟环丁基芳基醚结构引入聚碳酸酯主链. 通过对 以不同比例的 $\mathrm{PFCB} 2 \mathrm{OH}$ 单体和双酚 $\mathrm{A}$ 单体(BPA)为原 料缩合聚合制备的一系列具有不同全氟环丁基芳基醚 含量的聚碳酸酯的性质进行比较, 结果显示在不降低聚 合物透明性的前提下，全氟环丁基芳基醚结构的引入可 以显著提高聚碳酸酯材料的热稳定性和疏水性，并使得 聚碳酸酯具有很好的分散度.

表 2 聚碳酸酯的接触角和热稳定性

Table 2 Contact angle and thermostability of polycarbonates

\begin{tabular}{cccccccc}
\hline Sample & {$[\mathrm{PFCB} 2 \mathrm{OH}]:[\mathrm{BPA}]$} & Contact angle $/\left({ }^{\circ}\right)$ & $T^{\mathrm{a}} / \%$ & $T_{\mathrm{g}}^{b} /{ }^{\circ} \mathrm{C}$ & $T_{\mathrm{d}}^{c} /{ }^{\circ} \mathrm{C}$ & $T_{\mathrm{d}}^{c}(5 \%) /{ }^{\circ} \mathrm{C}$ & $T_{\mathrm{d}}^{c}(10 \%) /{ }^{\circ} \mathrm{C}$ \\
\hline PFCBPC & $1: 0$ & 110 & 100 & 130 & 351 & 392 & 412 \\
PFCBPC1 & $1: 1$ & 89 & 99 & 101 & 316 & 385 & 407 \\
PFCBPC2 & $1: 2$ & 83 & 100 & 103 & 310 & 380 & 397 \\
PFCBPC3 & $1: 3$ & 80 & 99 & 103 & 310 & 370 & 392 \\
PC & $0: 1$ & 50 & 95 & 121 & 308 & 374 & 396 \\
\hline
\end{tabular}

${ }^{a}$ The transmittance of the polymer in visible light; ${ }^{b} T_{\mathrm{g}}$ represents the glass transition temperature of the polymer under a nitrogen atmosphere; ${ }^{c} T_{\mathrm{d}}, T_{\mathrm{d}}(5 \%)$ and $T_{\mathrm{d}}$ $(10 \%)$ represent the decomposition temperature, the temperature of $5 \%$ mass loss and the temperature of $10 \%$ mass loss of the polymer under nitrogen atmosphere, respectively. 


\section{3 实验部分}

\section{1 实验原料}

三光气( $98 \%)$, 梯希爱; 对羟基苯甲醚(99\%), 锌粉, 阿拉丁; 碳酸铯, 麦克林; 1,2-二溴四氟乙烷 $(99 \%)$, 三 溴化嗍(99.99\%), 阿达玛斯; 四丁基溴化铵(99\%), 双酚 A $(96 \%)$, 北京百灵威科技有限公司. 所用试剂均从国 药集团化学试剂有限公司购买. 化合物 PFCB2OMe 按 已报道的方法合成 ${ }^{[13]}$.

\section{2 测试方法和仪器}

核磁共振采用的是 JEOL ECZ 400S 型核磁共振仪 测定, 氛代试剂为気代氯仿和気代二甲基亚砜, 氢谱的 内标为四甲基硅烷(TMS), 氟谱的外标为三氟乙酸; 红 外光谱(FT-IR)采用 Nicolet AVATAR-360 傅立叶变换红 外光谱仪 ( $\mathrm{KBr}$ 压片); 热失重分析(TGA)采用 TA Discovery TGA 55 热分析仪, 在 $\mathrm{N}_{2}$ 中测试, 加热速率为 $10{ }^{\circ} \mathrm{C} / \mathrm{min}$; 分子量采用 Waters 1515 凝胶色谱仪, 四氢 呋喃为淋洗剂, 线性聚苯乙烯为标准样品; 紫外可见光 谱采用 Hitachi U-2910 分光光度计; 差式扫描量热分析 (DSC)采用 TA Discovery DSC 250 差示扫描量热仪, 在 $\mathrm{N}_{2}$ 中测试, 加热速率为 $10{ }^{\circ} \mathrm{C} / \mathrm{min}$; 接触角测量采用 XG-CAMB1 静态接触角测量仪.

\section{3 单体 PFCB2OH 的合成}

对 $250 \mathrm{~mL}$ 装有滴液漏斗和抽气头的三口瓶进行㬌 抽一煤气焰烘烤一充氮气, 循环三次, 冷却至室温. 向三 口瓶中加入 PFCB2OMe $(46.0 \mathrm{~g}, 0.113 \mathrm{~mol})$ 和二氯甲烷 $(150 \mathrm{~mL})$, 摚拌 $10 \mathrm{~min}$. 用循环水泵将体系温度降低至 $0{ }^{\circ} \mathrm{C}$, 搅拌下将 $\mathrm{BBr}_{3}(25 \mathrm{~mL}, 0.226 \mathrm{~mol})$ 分批滴入三口 瓶, 用时 $30 \mathrm{~min}$. 滴加完毕后混合物升温至室温, 搅拌 3 $\mathrm{h}$, 加入 $250 \mathrm{~mL}$ 去离子水, 用 $150 \mathrm{~mL}$ 乙酸乙酯萃取 3 次, 除去有机溶剂得到 $40 \mathrm{~g}$ 灰色黏性固体, 产率 $93 \%$. m.p. $104{ }^{\circ} \mathrm{C} ;{ }^{1} \mathrm{H}$ NMR (DMSO- $\left.d_{6} 400 \mathrm{MHz}\right) \delta: 6.79$ (d, $J=6.84 \mathrm{~Hz}, 2 \mathrm{H}), 7.03$ (t, $J=6.84 \mathrm{~Hz}, 4 \mathrm{H}), 9.61(\mathrm{~d}, J=$ $7.90 \mathrm{~Hz}, 4 \mathrm{H}$ ); ${ }^{19} \mathrm{~F}$ NMR (DMSO- $\left.d_{6}, 400 \mathrm{MHz}\right) \delta$ : 128.08 - 132.51; FT-IR (KBr) v: 3524, 2363, 2332, $1660,1500,1185,961 \mathrm{~cm}^{-1}$; HRMS calcd for $\mathrm{C}_{16} \mathrm{H}_{10} \mathrm{O}_{4} \mathrm{~F}_{6}$ 380.22 , found 380.00 .

\section{4 聚碳酸酯的合成}

在 $100 \mathrm{~mL}$ 三口烧瓶中, 加入一定体积的 $1 \mathrm{~mol} / \mathrm{L}$ $\mathrm{NaOH}$ 的去离子水溶液, 之后加入等体积的溶有 1 equiv. 单体 (不同摩尔比的 PFCB2OH 和 BPA)的二氯甲烷溶液、 转子以及 0.05 equiv. 四丁基溴化铵. 将体系置于 $0{ }^{\circ} \mathrm{C}$ 冰水浴中保持 $20 \mathrm{~min}$ 后, 开始滴加二倍体积的含有 1.2 equiv.三光气的二氯甲烷溶液, 同时开始滴加 1.4 equiv.
三乙胺(当二氯甲烷溶液滴加 $2 / 3$ 体积时, 加入 $3 / 10$ 体积 的三乙胺; 当二氯甲烷溶液滴加结束时再滴加 $3 / 10$ 体积 的三乙胺; 最后反应 $30 \mathrm{~min}$ 后将剩余的三乙胺加入体 系). 滴加结束后，使体系自然恢复至室温，反应过夜. 反应停止后, 用去离子水萃取有机相, 直至水中无 $\mathrm{Cl}^{-}$ (硝酸银去检验). 之后除去溶剂, 用四氢呋喃溶解残余 物，在冰甲醇中沉降，得到一系列白色聚合物.

PFCBPC: 产率 $83 \%$. ${ }^{1} \mathrm{H}$ NMR $\left(\mathrm{CDCl}_{3}, 400 \mathrm{MHz}\right) \delta$ : $7.11 \sim 7.25(\mathrm{~m}, 8 \mathrm{H}) ;{ }^{19} \mathrm{~F} \mathrm{NMR}\left(\mathrm{CDCl}_{3}, 400 \mathrm{MHz}\right) \delta:-$ 126.39 -132.20; FT-IR (KBr) v: 3060, 1780, 1600, 1500, 1166, 961, $833 \mathrm{~cm}^{-1}$. GPC: $M_{n}=12500, M_{w} / M_{n}=$ 1.33 .

PFCBPC1: 产率 $86 \% .{ }^{1} \mathrm{H}$ NMR $\left(\mathrm{CDCl}_{3}, 400 \mathrm{MHz}\right)$ $\delta: 1.63 \sim 1.66(\mathrm{~m}, 6 \mathrm{H}), 7.15 \sim 7.38(\mathrm{~m}, 16 \mathrm{H}) ;{ }^{19} \mathrm{~F}$ NMR $\left(\mathrm{CDCl}_{3}, 400 \mathrm{MHz}\right) \delta:-126.64 \sim-131.45$; FT-IR $(\mathrm{KBr})$ $v: 2969,2364,2334,1778,1515,1162,1012,957 \mathrm{~cm}^{-1}$. GPC: $M_{n}=11600, M_{\mathrm{w}} / M_{\mathrm{n}}=1.37$.

PFCBPC2: 产率 78\%. ${ }^{1} \mathrm{H}$ NMR $\left(\mathrm{CDCl}_{3}, 400 \mathrm{MHz}\right)$ $\delta: 1.63 \sim 1.66(\mathrm{~m}, 6 \mathrm{H}), 7.14 \sim 7.25(\mathrm{~m}, 16 \mathrm{H}) ;{ }^{19} \mathrm{~F}$ NMR $\left(\mathrm{CDCl}_{3}, 400 \mathrm{MHz}\right) \delta:-128.62 \sim-131.88$; FT-IR $(\mathrm{KBr})$ $v: 3080,2363,2345,1783,1506,1160,962 \mathrm{~cm}^{-1}$. GPC: $M_{\mathrm{n}}=16200, M_{\mathrm{w}} / M_{\mathrm{n}}=1.41$.

PFCBPC3: 产率 74\%. ${ }^{1} \mathrm{H}$ NMR $\left(\mathrm{CDCl}_{3}, 400 \mathrm{MHz}\right)$ $\delta: 1.63 \sim 1.67(\mathrm{~m}, 6 \mathrm{H}), 7.14 \sim 7.25(\mathrm{~m}, 16 \mathrm{H})$; FT-IR $(\mathrm{KBr})$ $v: 2969,2363,2339,1778,1500,1148,1018,962 \mathrm{~cm}^{-1}$. GPC: $M_{\mathrm{n}}=15400, M_{\mathrm{w}} / M_{\mathrm{n}}=1.43$.

PC: 产率 $82 \% .{ }^{1} \mathrm{H}$ NMR $\left(\mathrm{CDCl}_{3}, 400 \mathrm{MHz}\right) \delta$ : $1.63 \sim 1.66(\mathrm{~m}, 6 \mathrm{H}), 7.14 \sim 7.25(\mathrm{~m}, 16 \mathrm{H})$; FT-IR $(\mathrm{KBr}) v$ : 2969, 2363, 2332, 1778, 1512, 1191, 1093, $827 \mathrm{~cm}^{-1}$. GPC: $M_{\mathrm{n}}=12500, M_{\mathrm{w}} / M_{\mathrm{n}}=1.46$.

辅助材料(Supporting Information) 单体 PFCB2OH 及 聚合物 PFCBPC, PFCBPC1, PFCBPC2, PFCBPC3, PC 的 ${ }^{1} \mathrm{H}$ NMR、 ${ }^{19} \mathrm{~F}$ NMR、FT-IR 谱图及 GPC 曲线. 这些材料 可以免费从本刊网站(http://sioc-journal.cn/)上下载.

\section{References}

[1] Jang, M.; Chan, K. P.; Lee, N. Y. Sens. Actuators, B 2014, 193, 599.

[2] Seubert, C.; Nietering, K.; Nichols, M.; Wykoff, R.; Bollin, S. Coatings 2012, 2, 221

[3] Choi, M. C.; Kim, Y.; Ha, C. S. Prog. Polym. Sci. 2008, 33, 581

[4] Lahijania, Y. Z. K.; Mohseni, M.; Bastani, S. Tribol. Int. 2014, 69, 10.

[5] Liu, Z.; Zhang, X.; Murakami, T.; Fujishima, A. Sol. Energy Mater. Sol. Cells 2008, 92, 1434.

[6] Hu, D.-B. M.S. Dissertation, Wuhan University of Technology, Wuhan, 2013 (in Chinese).

[7] Shirtcliffe, N. J.; Mchale, G.; Newton, M. I. J. Polym. Sci., Part B: Polym. Phys. 2011, 49, 1203.

[8] Liu, X.; Dai, B.; Zhou, L.; Sun, J. J. Mater. Chem. 2009, 19, 497. 
[9] Youngblood, J. P.; McCarthy, T. J. Macromolecules 1999, 32, 6800

[10] Teshima, K.; Sugimura, H.; Inoue, Y.; Takai, O.; Takano, A. Langmuir 2003, 19, 10624.

[11] Zheng, Z.-L. M.S. Thesis, Hefei University of Technology, Hefei, 2013 (in Chinese) (郑正龙，硕士论文，合肥工业大学，合肥, 2013.)

[12] Pagliaro, M.; Ciriminna, R. J. Mater. Chem. 2005, 15, 4981.

[13] Babb, D. A.; Ezzell, B. R.; Clement, K. S.; Richey, W. F.; Kennedy, A. P. J. Polym. Sci., Part A: Polym. Chem. 1993, 31, 3465.

[14] Rizzo, J.; Harris, F. W. Polymer 2000, 41, 5125.

[15] Liu, S.; Jiang, X.; Ma, H.; Liu, M. S.; Jen, A. K. Y. Macromolecules 2000, 33, 3514 .

[16] Jin, J.; Smith, D. W.; Topping, C. M.; Suresh, S.; Chen, S.; Foulger, S. H.; Rice, N.; Nebo, J.; Mojazza, B. H. Macromolecules 2003, 36,
9000.

[17] Ma, H.; Wu, J.; Herguth, P.; Chen, B.; Jen, A. K. Y. Chem. Mater. 2000, 12, 1187.

[18] Corley, C. A.; Guenthner, A. J.; Sahagun, C. M.; Lamison, K. R.; Reams, J. T.; Hassan, M. K.; Morgan, S. E.; Iacono, S. T.; Mabry, J. M. ACS Macro Lett. 2014, 3, 105.

[19] Li, Y.; Zhang, S.; Tong, L.; Li, Q.; Li, W.; Lu, G.; Liu, H.; Huang, X. J. Fluorine Chem. 2009, 130, 354.

[20] Jia, M.; Zhou, M.; Li, Y.; Lu, G.; Huang, X. Polym. Chem. 2018, 9 , 920.

[21] Spraul, B. K.; Suresh, S.; Jin, J.; Smith, D. W. J. Am. Chem. Soc. 2006, 128, 7055

[22] Yuan, C.; Wang, J.; Jin, K.; Diao, S.; Sun, J.; Tong, J.; Fang, Q. Macromolecules 2014, 47, 6311.

(Zhao, X.) 